\title{
Indicadores de impacto social en pacientes diagnosticados de catarata senil tratados con facoemulsificación más implante de lente intraocular
}

\author{
Social impact indicators in patient diagnosed of senile cataract treated with \\ phacoemulsification plus intraocular lens implantation
}

\author{
Perla K. Onofre-Déciga ${ }^{1 *}$, Claudia Palacio-Pastrana ${ }^{1}$, Cyntia Solís-Hernández y Héctor Pérez-Cano ${ }^{2}$ \\ ${ }^{1}$ Departamento de Segmento Anterior; ${ }^{2}$ Centro de Investigación Biomédica. Fundación Hospital Nuestra Señora de la Luz, Ciudad de México, \\ México
}

\section{Resumen}

Objetivo: Analizar indicadores de calidad de vida en pacientes con catarata senil tratados con facoemulsificación más implante de lente intraocular. Método: Pacientes a partir de 60 años de edad, con agudeza visual mejor corregida (AVMC) $\geq 0.6$ LogMAR y catarata senil tratada con facoemulsificación más implante de lente intraocular para casos y catarata senil incipiente sin tratamiento quirúrgico con AVMC < 0.6 LogMAR para controles. Criterios de exclusión: patologías que modificaran la calidad de vida. Se aplicó la encuesta WHOQOL-OLD en el preoperatorio y a 1 y 3 meses posquirúrgicos. Resultados: 54 controles y 56 casos. La AVMC preoperatoria fue $1.57 \pm 1.041$, y al tercer mes $0.68 \pm 0.7817$ ( $<<0.001)$. Los resultados de la encuesta preoperatoria en comparación con el tercer mes fueron: dominio de intimidad 12.96 vs. 11.06 ( $p<0.0001)$; dominio de muerte y agonía 10.48 vs. 6.389 ( $p<0.00001$ ); dominio de participación social 12.07 vs. 13.76 ( $p<0.0007)$; y dominio de capacidad sensorial 13.02 vs. 8.648 ( $p<0.0001)$. Los resultados de la encuesta del tercer mes en comparación con los controles fueron: dominio de intimidad 9.66 vs. 11.06 ( $p<0.001)$; dominio de actividades pasadas, presentes y futuras 13.89 vs. 12.39 ( $p$ < 0.001) y dominio de autonomía 12.47 vs. $10.15(p<0.001)$. Conclusión: Los pacientes presentan una mejora en la AVMC y en los resultados de la WHOQOL-OLD, por lo que deben ser tomados en cuenta para apoyar la indicación quirúrgica.

Palabras Clave: Adultos mayores. Agudeza visual. Calidad de vida. Facoemulsificación. WHOQOL-OLD.

\begin{abstract}
Purpose: To analyze indicators of social impact in patients with senile cataract treated with phacoemulsification and intraocular lens (IOL) implant. Material and methods: Patients $\geq 60$ years, better corrected visual acuity (BCVA) $\geq 0.6$ logarithm of the minimum angle of resolution (LogMAR) and senile cataract treated with PHACO + IOL for cases and incipient senile cataract without surgical treatment with MVCA < 0.6 LogMAR for controls. Exclusion criteria; pathologies that modify the quality of life. The WHOQOL-OLD test was applied: presurgical, one postoperative month and three postoperative months. Results: 54 controls and 56 cases were included. The preoperative BCVA was $1.57 \pm 1.041$, third month was $0.68 \pm 0.7817(p<0.001)$. The results of the preoperative WHOQOL-OLD survey vs the third month: INT 12.96 versus $11.06(p<0001) ; D A D, 10.48$
\end{abstract}

\section{Correspondencia:}

*Perla K. Onofre-Déciga

Ezequiel Montes, 135

Col. Tabacalera, Del. Cuauhtémoc

C.P. 06030, Ciudad de México, México

Fecha de recepción: 12-06-2019

Cir Cir. 2020;88(3):331-336

E-mail: onodec1@gmail.com

Fecha de aceptación: 25-11-2019

DOI: $10.24875 / C I R U .19001372$

Contents available at PubMed

www.cirugiaycirujanos.com

0009-7411/@ 2019 Academia Mexicana de Cirugía. Publicado por Permanyer. Este es un artículo open access bajo la licencia CC BY-NC-ND (http://creativecommons.org/licenses/by-nc-nd/4.0/). 
versus 6.389 ( $p<0.00001)$; SOP 12.07 versus $13.76(p<0.0007)$ and SAB, 13.02 versus $8.648(p<0.0001)$. The survey of the $3^{\text {rd }}$ month versus the controls: INT 9.66 versus $11.06(p<0.001)$; PPF, 13.89 versus $12.39(p<0.001)$ and AUT 12.47 versus $10.15(p<0.001)$. Conclusion: Patients, present an improvement in the BCVA that is reflected in the quality of life, this can be interpreted as positive results and should be taken into account to support the surgical indication.

Key Words: Older adults. Phacoemulsification. Quality of life. Visual acuity. WHOQOL-OLD.

\section{Introducción}

La catarata senil es una enfermedad de alta prevalencia mundial que afecta la visión de forma parcial o total debido a una opacidad progresiva del cristalino. Es la principal causa de ceguera en todo el mundo, por lo que su detección oportuna y tratamiento son el manejo ideal ${ }^{1,2}$. Sin embargo, lo anterior puede ser poco viable en países en vías de desarrollo o en comunidades carentes de atención médica oportuna y de calidad, donde el tratamiento quirúrgico no se realiza ${ }^{2,3}$.

En el mundo hay aproximadamente 285 millones de personas con discapacidad visual, de las cuales 39 millones son ciegas y 246 millones presentan baja visión ${ }^{4}$. Cerca de un $90 \%$ de la discapacidad visual mundial se concentra en los países emergentes ${ }^{2}$. En los países de ingresos medios y bajos, las cataratas siguen siendo la principal causa de ceguera ${ }^{3}$. El $82 \%$ de las personas con ceguera tienen 50 años o más, y cerca del $80 \%$ del total mundial de casos de discapacidad visual podrían evitarse o ser susceptibles de tratamiento ${ }^{4,5}$.

En México, varios grupos de estudio han realizado investigaciones en el norte y sur del país ${ }^{3}$. En el Estado de Nuevo León se identificó una prevalencia de ceguera en personas mayores de 50 años del $1.53 \%$, y el $48 \%$ de estas cegueras fueron atribuibles a cataratas. En el Estado de Chiapas se identificó una prevalencia de ceguera en personas de 50 años o más del $2.3 \%$ y el $63 \%$ de estas cegueras fueron atribuibles a cataratas ${ }^{6}$.

La presencia de catarata senil ocasionará baja visual indolora progresiva. Conforme la visión disminuye, los pacientes presentan restricciones para realizar ciertas actividades de la vida diaria, como la preparación de alimento, la higiene personal y la lectura ${ }^{7,8}$. De forma paralela, el individuo tiene más riesgo de tener pérdida del equilibrio, caídas, fracturas o cualquier otro tipo de lesiones físicas. Todo esto puede repercutir en el bienestar mental y físico de la persona ${ }^{9}$. El médico debe indagar sobre estos signos, ya que en ellos podrían recaer las indicaciones de tratamiento quirúrgico.
El tratamiento de las cataratas seniles actualmente es quirúrgico; esta cirugía, gracias a los avances biomédicos de la microcirugía oftalmológica, se ha ganado su lugar como pilar en el tratamiento de las cataratas $^{10}$. Es de gran importancia recalcar que no existe ningún tratamiento médico en nuestros días que haya probado reducir el tamaño, frenar la progresión o evitar la aparición de una catarata ${ }^{11}$.

La indicación ampliamente usada para la cirugía de cataratas es la disminución de la agudeza visual funcional para el paciente que le impida al paciente un adecuado desempeño en su quehacer diario ${ }^{12}$. Es importante mencionar que existen pocas contraindicaciones de cirugía de cataratas en un adulto mayor, siempre y cuando existan unas condiciones sistémicas estables ${ }^{10,12}$.

Para los profesionales de la salud, la calidad de vida relacionada con la salud debe ser un aspecto indispensable a evaluar, por lo que el uso de instrumentos clinimétricos para su medición es una promesa en ámbitos como el apego al tratamiento, la satisfacción con los servicios brindados y la efectividad de las intervenciones médicas y de los programas de atención a la salud ${ }^{13,14}$. En México, los reportes de investigación dirigidos al estudio de la calidad de vida y su repercusión social son escasos ${ }^{15}$.

El cuestionario de evaluación de la calidad de vida de la Organización Mundial de la Salud (WHOQOL, World Health Organization Quality of Life) ha sido utilizado durante más de 35 años de manera efecti$\mathrm{va}^{16}$. Existen versiones derivadas de este cuestionario, una de las cuales es el cuestionario para adultos mayores (WHOQOL-OLD) ${ }^{17}$.

En México, la encuesta WHOQOL-OLD ya ha sido validada, obteniendo así propiedades psicométricas de confiabilidad y validez ${ }^{18,19}$. Esta encuesta se compone de 24 preguntas de opción múltiple que estudian seis dominios. El dominio de intimidad se refiere a las experiencias del adulto mayor en relación con su sentido de compañerismo y sentimiento de amor recíproco con la gente más allegada. El dominio de muerte y agonía considera el sentimiento de miedo y la preocupación acerca de la forma y el tiempo de morir. El dominio de autonomía estima la capacidad 
y la libertad de los adultos mayores para tomar decisiones y control en su desarrollo personal y en su entorno cercano. El dominio de capacidad sensorial evalúa el impacto en la vida diaria de los adultos mayores respecto a la pérdida de habilidades sensoriales. El dominio de actividades pasadas, presentes y futuras estima la satisfacción con los logros cumplidos en la vida y los resultados que se esperan en las actividades futuras. El dominio de participación social se refiere a la participación en las actividades de la vida diaria, especialmente en la comunidad del adulto mayor. Todos los dominios se analizan por separado y se genera una puntuación final. Básicamente, cuanto mayores sean puntajes, mejor calidad de vida; hay que señalar que los dominios de capacidad sensorial, intimidad, y muerte y agonía, se codifican a la inversa ${ }^{20,21}$.

Según nuestro conocimiento, no existen investigaciones que hayan evaluado la calidad de vida en pacientes con diagnóstico de catarata sometidos a cirugía de facoemulsificacion más implante de lente intraocular en población mexicana. El objetivo del presente estudio fue identificar cambios en la calidad de vida, mediante el cuestionario WHOQOL-OLD, y en la agudeza visual en pacientes con diagnóstico de catarata senil sometidos a facoemulsificación más implante de lente intraocular.

\section{Método}

Se realizó un estudio descriptivo, prospectivo, de casos y controles, incluyendo dos grupos de pacientes: 1) pacientes que se sometieron a facoemulsificación más implante de lente intraocular y 2) pacientes con catarata senil incipiente que no fueron sometidos a cirugía.

Los criterios de inclusión fueron pacientes $\geq 60$ años en ambos grupos, con agudeza visual mejor corregida $(\mathrm{AVMC}) \geq 0.6$ LogMAR para el grupo de los casos $y<0.6$ LogMAR para el grupo control. Los criterios de exclusión fueron otras patologías oculares, enfermedades sistémicas y otras discapacidades sensoriales que modifiquen la calidad de vida. Para evaluar la calidad de vida se aplicó la encuesta WHOQOL-OLD, en el grupo de los casos en tres ocasiones: antes de la cirugía, 1 mes y 3 meses después de la operación, previa firma del consentimiento informado. La misma encuesta fue aplicada en el grupo control en una sola ocasión.

Para el análisis estadístico se utilizaron los programas Excel (Microsoft Office, 2018) y GraphPad Prism

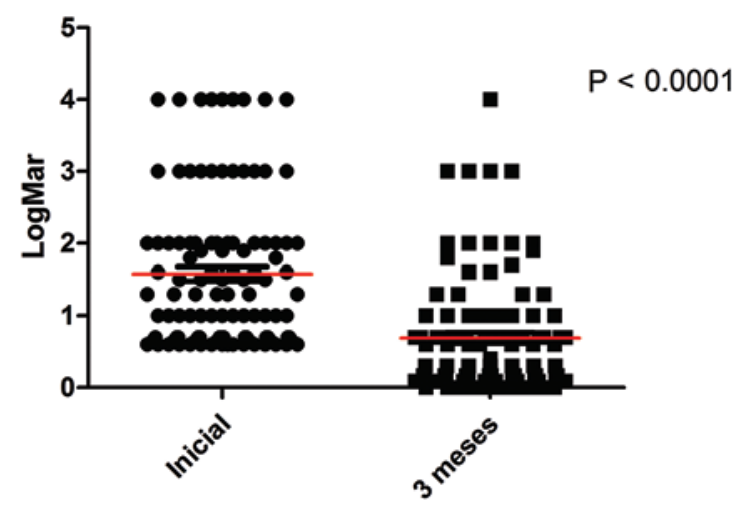

Figura 1. Comparación de la agudeza visual mejor corregida (AVMC) preoperatoria y a los 3 meses de la cirugía.

V5.0. Se realizó la prueba de ANOVA de una vía para la comparación de los seis dominios de la encuesta al inicio y a los 3 meses posoperatorios en el grupo de casos, y una prueba de ANOVA de una vía para la comparación de los seis dominios medidos por la encuesta en el tercer mes posoperatorio y la encuesta aplicada al grupo control. En aquellos en los que se encontró una diferencia significativa se utilizó la prueba de Mann Withney para comparar por dominio. Se consideró una $p<0.05$ como estadísticamente significativa.

\section{Resultados}

Se reclutaron 108 personas: 54 casos y 56 controles. La edad promedio fue de 72 años para los pacientes y de 68 años para los sujetos sanos. El $63 \%$ de las personas encuestadas eran mujeres y el $37 \%$ eran hombres, en ambos grupos. La AVMC preoperatoria fue de $1.57 \pm 1.041$ LogMAR y en el tercer mes fue de $0.68 \pm 0.7817$ LogMar $(p<0.001)$ (Fig. 1).

Los resultados comparativos de los dominios de las encuestas preoperatoria y del primer mes se muestran en la tabla 1. En la tabla 2 se muestran los resultados comparativos de los dominios de las encuestas preoperatoria y del tercer mes. Los resultados comparativos de los dominios de las encuestas aplicadas al grupo de los casos en el tercer mes y al grupo de los controles se muestran en la tabla 3.

\section{Discusión}

Anteriormente, el resultado de una cirugía de catarata solo se evaluaba con la medición de la agudeza visual, pero con el paso del tiempo, la autopercepción 
Tabla 1. Resultados de los seis dominios de la encuesta preoperatoria y a los 2 meses posoperatorios

\begin{tabular}{lccc}
\hline Dominios & \multicolumn{2}{c}{ Casos } & p \\
\cline { 2 - 3 } & $\begin{array}{c}\text { Encuesta } \\
\text { preoperatoria }\end{array}$ & $\begin{array}{c}\text { Encuesta } \\
\text { 2 meses }\end{array}$ & \\
\hline Participación social & 12.07 & 12.44 & NS \\
Intimidad & 12.96 & 13.20 & NS \\
Muerte y agonía & 10.48 & 11.76 & 0.0254 \\
Capacidad sensorial & 13.02 & 12.22 & 0.0033 \\
Autonomía & 10.67 & 11.89 & 0.0167 \\
Actividades pasadas, & 12.72 & 12.80 & NS \\
presentes y futuras & & & \\
\hline NS: no significativo. & & &
\end{tabular}

Tabla 2. Resultados de los seis dominios de la encuesta preoperatoria y a los 3 meses posoperatorios

\begin{tabular}{lccc}
\hline Dominios & \multicolumn{2}{c}{ Casos } & p \\
\cline { 2 - 3 } & $\begin{array}{c}\text { Encuesta } \\
\text { preoperatoria }\end{array}$ & $\begin{array}{c}\text { Encuesta } \\
\text { 3 meses }\end{array}$ & \\
\hline Participación social & 12.07 & 13.76 & $<0.0007$ \\
Intimidad & 12.96 & 11.06 & $<0.0001$ \\
Muerte y agonía & 10.48 & 6.38 & $<0.00001$ \\
Capacidad sensorial & 13.02 & 8.64 & $<0.0001$ \\
Autonomía & 10.67 & 10.15 & NS \\
Actividades pasadas, & 12.72 & 12.39 & NS \\
presentes y futuras & & & \\
\hline NS: no significativo. & & &
\end{tabular}

Tabla 3. Resultados de los seis dominios de la encuesta a los 3 meses posoperatorios en el grupo de los casos y de la encuesta aplicada a los controles

\begin{tabular}{lccc}
\hline Dominios & $\begin{array}{c}\text { Encuesta 3 } \\
\text { meses casos }\end{array}$ & $\begin{array}{c}\text { Encuesta } \\
\text { controles }\end{array}$ & $\mathbf{p}$ \\
\hline Participación social & 13.76 & 13.84 & NS \\
Intimidad & 11.06 & 9.66 & $<0.0001$ \\
Muerte y agonía & 6.38 & 5.89 & NS \\
Capacidad sensorial & 8.64 & 8.59 & NS \\
Autonomía & 10.15 & 12.47 & $<0.0001$ \\
Actividades pasadas, & 12.39 & 13.89 & $<0.0001$ \\
presentes y futuras & & & \\
\hline NS: no significativo. & & &
\end{tabular}

y las repercusiones en la vida diaria del paciente han ganado terreno ${ }^{22}$. Nuestro estudio midió los indicadores de calidad de vida en los pacientes sometidos a facoemulsificación más implante de lente intraocular, y se encontró que los pacientes sometidos a cirugía de catarata tienen una mejora en la calidad de vida y en la agudeza visual, basándose en la respuesta clínica favorable que se obtuvo a través de la encuesta WHOQOL-OLD y de la cartilla de Snellen. Se puede comparar este resultado con estudios previos que, usando herramientas clínimétricas diferentes, obtuvieron una mejoría en la calidad de vida ${ }^{23,24}$. Nuestro seguimiento fue de 3 meses posoperatorios, ya que es tiempo suficiente para valorar una respuesta favorable en estos dos indicadores. Existen investigaciones previas con seguimiento de 3 meses que muestran una mejoría en la función visual y en la calidad de vida, y hay así mismo algunos otros estudios con seguimiento de 7 meses en los cuales se alcanzó un impacto en todos los aspectos de la vida, inclusive en la calidad del sueño ${ }^{25}$.

La comparación entre los resultados obtenidos en la encuesta realizada en los tres tiempos diferentes en el grupo de los casos muestra una clara mejoría a los 90 días posoperatorios. Esto se puede explicar por la ausencia de necesidad de ser cuidados por terceras personas, por un aumento de la independencia y por un mayor control de la vida de los pacientes en el posoperatorio mediato.

Por otro lado, la mejora en la agudeza visual que obtuvieron los pacientes en el grupo de los casos, a los 3 meses, concuerda con lo publicado por Bandhu et al. ${ }^{26}$, al encontrar una mejoría del $99 \%$ en la visión posoperatoria.

A pesar de los excelentes resultados visuales, no pudimos observar esta misma mejoría reflejada en el $100 \%$ de los dominios que se examinaron, al igual que lo previamente reportado por Schein et al. ${ }^{27}$, quienes justificaron este resultado por la comorbilidad oftalmológica en la gran mayoría de sus pacientes (glaucoma, uveítis o degeneración macular relacionada con la edad, por mencionar algunos ejemplos). En el presente estudio se excluyeron los pacientes que presentaban otras patologías oftalmológicas o sistémicas que afecten a la visión o a la calidad de vida. La ausencia de mejoría total en los dominios puede explicarse porque algunos de ellos, como la participación social, la autonomía o las actividades pasadas, presentes y futuras, no están relacionados solo con el padecimiento oftalmológico, pues pueden resultar afectados por factores como la edad, las altas expectativas de los pacientes, la falla de la red de apoyo familiar o situaciones socioeconómicas imposibles de mejorar ${ }^{28}$.

Dentro de las limitaciones de nuestro estudio se encuentra el tamaño de la muestra; no obstante, a 
pesar de ser pequeña, fue posible realizar un análisis estadístico que permitió obtener resultados confiables y equiparables a los de otros estudios publicados. Otras posibles limitaciones son la utilización de cuestionarios para la medición de la función visual y la evaluación con el cuestionario de calidad de vida para una valoración integral del impacto de la cirugía de catarata. Resultaría interesante realizar un estudio estratificando a los pacientes por otros factores que afectan la calidad de vida, para evitar un posible sesgo de membresía.

Pese a las limitaciones mencionadas, los indicadores que se midieron en el presente estudio (calidad de vida y AVMC) mostraron una mejoría desde el tercer mes posoperatorio. Estos resultados no son solo relevantes para el paciente, sino también para todo el equipo médico involucrado en cada cirugía de catarata, ya que dan pie a mejorar su actuar frente a la catarata senil al utilizarlos como parte de la evaluación preoperatoria habitual. Lee y $\mathrm{Kymes}^{29}$ concluyeron que la cirugía de catarata debe ser considerada como uno de los tratamientos quirúrgicos con una mayor relación costo-efectividad.

Debe señalarse que los resultados obtenidos han sido comparados con estudios publicados en países desarrollados y demuestran la utilidad de la facoemulsificación más implante de lente intraocular como tratamiento de elección de las cataratas, brindando una recuperación pronta que genera un impacto positivo en la calidad de vida de los pacientes.

\section{Conclusión}

La calidad de vida es un concepto tan amplio que no puede ajustarse a la explicación de un solo aspecto o punto de vista. Los pacientes con catarata senil sometidos a facoemulsificación más implante de lente intraocular presentan una mejora en la AVMC que se refleja en la calidad de vida; esto puede ser interpretado como resultados positivos y deben ser tomados en cuenta para apoyar la indicación quirúrgica. El uso de herramientas clinimétricas debe incluirse en la práctica diaria del médico oftalmolólogo para la atención integral de los pacientes.

\section{Agradecimientos}

Al Dr. Jaime Lozano Alcázar y la Dra. Adriana Saucedo Castillo.

\section{Conflicto de intereses}

Todos los autores declaran no tener conflictos de intereses.

\section{Responsabilidades éticas}

Protección de personas y animales. Los autores declaran que para esta investigación no se han realizado experimentos en seres humanos ni en animales.

Confidencialidad de los datos. Los autores declaran que han seguido los protocolos de su centro de trabajo sobre la publicación de datos de pacientes.

Derecho a la privacidad y consentimiento informado. Los autores han obtenido el consentimiento informado de los pacientes y/o sujetos referidos en el artículo. Este documento obra en poder del autor de correspondencia.

\section{Bibliografía}

1. Thylefors B, Négrel AD, Pararajasegaram R, Dadzie KY. Global data on blindness. Bull World Health Organ. 1995;73:115-121.

2. Ono K, Hiratsuka Y, Murakami A. Global inequality in eye health: country-level analysis from the global burden of disease study. Am J Public Health. 2010;100:1784-8.

3. Bastar PAG, Lansingh VC, Penniecook-Sawyers JA, et al. La catarata sigue siendo la principal causa de ceguera en economías emergentes, incluyendo México. Rev Mex Oftalmol. 2014;88:208-9.

4. World Health Organization. Ceguera y discapacidad visual. 2018. (Consultado el 2 de noviembre de 2018.) Disponible en: https:/www.who.int/ es/news-room/fact-sheets/detail/blindness-and-visual-impairment

5. World Health Organization. Salud ocular universal: un plan de acción mundial 2014-2019. 2013. Disponible en: https://www.who.int/blindness/ AP2014 19 Spanish.pdf?ua=1

6. Visión 2020. Situación actual de ceguera por catarata en México. 2015. (Consultado el 2 de noviembre de 2018.) Disponible en: https://vision2020la.wordpress.com/2015/01/15/situacion-actual-de-ceguera-por-catarata-en-mexico

7. Bowling B, Kanski JJ. Kanski's Clinical ophtalmology: a systematic approach. Edinburgh: Elsevier; 2015.

8. Asbell P, Dualan I, Mindel J, Brocks D, Ahmad M, Epstein S. Age-related cataract. Lancet. 2005;365:599-609.

9. Jimenez A, Jimenez ME, Ponce De Leon S, Chavez M, Graue EO. Social determinants and their impact on visual impairment in Southern Mexico. Ophthalmic Epidemiol. 2014;22:342-8

10. Syam PP, Eleftheriadis H, Casswell AG, Brittain GP, Mcleod BK, Liu CSC. Clinical outcome following cataract surgery in very elderly patients. Eye. 2004;18:59-62

11. Lundström M, Stenevi U, Thorburn W. Cataract surgery in the very elderly. JCRS. 2000;26:408-14.

12. Bass EB, Wills S, Scott IU, Javitt JC, Tielsch JM, Schein OD, et al. Preference values for visual states in patients planning to undergo cataract surgery. Med Decis Making. 1997;17:324-30.

13. Testa MA, Simonson DC. Assessment of quality-of-life outcomes. N Engl Jed. 1996;334:835-40.

14. Salvador R, Romero $H$. Reflexiones sobre calidad de vida relacionada con la salud. Rev Med Inst Mex Seguro Soc. 2010;48:91-102.

15. Velarde-Jurado E, Ávila-Figueroa C. Evaluación de la calidad de vida. Salud Publica Mex. 2002;44:349-61.

16. World Health Organization. Division of Mental Health and Prevention of Substance Abuse. WHOQOL: measuring quality of life. World Health Organization; 1997ロ. Disponible en: https://apps.who.int/iris/handle/10665/63482

17. González AL, Gómez J. Quality of life in the elderly: psychometric properties of the WHOQOL-OLD module in Mexico. Health. 2013:5:110-6.

18. Acosta CO, Vales JJ, Echeverría SB, Serrano DM, García R. Confiabilidad y validez del Cuestionario de Calidad de Vida (WHOQOL-OLD) en adultos mayores mexicanos. Psicología y Salud. 2013;23:241-50. 
Cirugía y Cirujanos. 2020;88(3)

19. Hernández JC, Guadarrama R, Castillo S, Arzate G, Márquez O. Validación del WHOQOL-OLD en adultos mayores de México. Revista Latinoamericana de Ciencia Psicológica. 2015;(7):397-405.

20. The World Health Organization Quality Of Life assessment (WHOQOL): position paper from the World Health Organization. Soc Sci Med 1995;41:1403-9.

21. World Health Organization. Programme on mental health: WHOQOL use manual, 2012 revision. Disponible en: https://apps.who.int/iris/handle/10665/77932

22. Bernth-Petersen P. Visual functioning in cataract patients. Acta Ophthalmol. 2009;59:198-205.

23. Mamidipudi PR, Vasavada AR, Merchant SV, Namboodiri V, Ravilla TD Quality-of-life and visual function assessment after phacoemulsification in an urban Indian population. J Cataract Refract Surg. 2003 29:1143-51.
24. Paredes SL, Malqui OP, Duglio MA, Tito MV, Mayta-Tristán P. Variación de la función visual y calidad de vida luego de cirugía de catarata por facoemulsificación con implante de lente intraocular. Rev Mex Oftalmol. 2014;88:176-81.

25. Ayaki M, Muramatsu M, Negishi K, Tsubota K. Improvements in sleep quality and gait speed after cataract surgery. Rejuvenation Res. 2013;16:35-42.

26. Bandhu S, Vabale Y, Sambarey P, Walke A, Raje S. Impact of cataract on the quality of life of rural patients in India. Journal of Clinical Ophthalmology and Research. 2016;4:75.

27. Schein OD, Steinberg EP, Cassard SD, Tielsch JM, Javitt JC, Sommer A Predictors of outcome in patients who underwent cataract surgery. Ophthalmology. 1995;102:817-9.

28. Morris D, Fraser SG, Gray C. Cataract surgery and quality of life implications. Clin Interv Aging. 2007;2:105-8.

29. Lee BS, Kymes SM. Cataract surgery cost utility revisited in 2012: a new economic paradigm. Ophthalmology. 2013;122:2367-76. 